Pacific

Journal of

Mathematics

FLAT MODULES AND LIFTING OF FINITELY GENERATED PROJECTIVE MODULES

Alberto Facchini, Dolors Herbera AND IsKhaK SAKHAJEV 


\title{
FLAT MODULES AND LIFTING OF FINITELY GENERATED PROJECTIVE MODULES
}

\author{
Alberto FACChini, Dolors Herbera AND ISKHAK SAKHAJEV
}

\begin{abstract}
We introduce nets in rings, which turn out to describe right flat modules and left flat modules over a fixed ring $R$ at the same time. As an application we prove that for a finitely generated projective right $R / J(R)$-module $P$, there exists a finitely generated flat right $R$-module $M$ with $M / M J(R)$ isomorphic to $P$ if and only if there exists a projective left $R$-module $P^{\prime}$ with $P^{\prime} / J(R) P^{\prime}$ isomorphic to the dual of $P$.
\end{abstract}

\section{Introduction}

Although there is a close relation between finitely generated projective right $R$ modules and finitely generated projective left $R$-modules given by the duality $\operatorname{Hom}_{R}(-, R)$, there does not seem to be such an evident relation between finitely generated flat right $R$-modules and finitely generated flat left $R$-modules. In this paper we define an algebraic object that allows us to describe right flat modules and left flat modules at the same time. We call this algebraic object a net, because its definition recalls the definition of nets encountered in topology. Our concept finds its origin in [Vasconcelos 1969, proof of Theorem 2.1], and was implicitly used in [Lazard 1974; Sakhaev 1987; 1993; 1996]. As an application of our theory, we study how projective modules over the ring $R / J(R)$ lift to projective or flat modules over $R$. For instance, we find that for a finitely generated projective right $R / J(R)$-module $P$, there exists a finitely generated flat right $R$-module $M$ with $M / M J(R)$ isomorphic to $P$ if and only if there exists a projective left $R$-module $P^{\prime}$ with $P^{\prime} / J(R) P^{\prime}$ isomorphic to the dual $\operatorname{Hom}_{R / J(R)}(P, R / J(R)$ ) of $P$ (Theorem 7.1).

MSC2000: 16D40.

Keywords: flat modules, projective covers.

The research of the first author was supported by Ministero dell'Istruzione, dell'Università e della Ricerca (Italy) and by Departament d'Universitats, Recerca i Societat de la Informació (Generalitat de Catalunya, Spain). This paper was written during a sabbatical year at the Centre de Recerca Matemàtica (Barcelona). The first author acknowledges the kind hospitality received. The research of the second author was partially supported by the DGI and the European Regional Development Fund, jointly, through Project BFM2002-01390, and by the Comissionat per Universitats i Recerca of the Generalitat de Catalunya. 
The paper is organized as follows. In the next two sections we give our basic definitions and constructions. We define nets in rings and show how it is possible to associate to each net both a flat cyclic right module and a flat cyclic left module. In Section 4 we prove that this construction allows us to describe all flat right or left modules.

In Section 5 we give a couple of examples. The first one is the flat module introduced in [Bass 1960]. The second one is based on [Sakhaev 1987; 1993; 1996] and is the key tool in the last two sections to study finitely generated flat modules that are projective modulo the Jacobson radical.

Our rings are associative and have an identity. Modules are unital. For every module $M_{R}$, we denote by $\mathscr{L}\left(M_{R}\right)$ the set of all submodules of $M_{R}$. The Jacobson radical of a ring $R$ is denoted by $J(R)$.

\section{Nets in rings}

In this section we introduce the concepts that will be used freely throughout the paper.

Let $A$ be a set with a transitive relation $<$. (We denote the relation by $<$, not $\leq$, to stress that it is not necessarily reflexive.)

Definition 2.1. A net in $A$ is a pair $(\Lambda, \varphi)$, where

(1) $\Lambda$ is a nonempty partially ordered set, without a greatest element, without a least element, and with $\Lambda$ upward directed and downward directed (that is, for each pair $\lambda, \mu$ in $\Lambda$ there exist $\nu$ and $\xi$ in $\Lambda$ such that $\lambda \leq \nu, \mu \leq \nu, \xi \leq \lambda$ and $\xi \leq \mu)$;

(2) $\varphi: \Lambda \rightarrow A$ is a strictly increasing map, that is, for every $\lambda, \mu \in \Lambda, \lambda \leq \mu$ and $\lambda \neq \mu$ implies $\varphi(\lambda)<\varphi(\mu)$.

For every $\lambda, \mu \in \Lambda$, we shall write $\lambda<\mu$ whenever $\lambda \leq \mu$ and $\lambda \neq \mu$. Whenever $(\Lambda, \varphi)$ is a net in $A$, we will usually write $a_{\lambda}$ instead of $\varphi(\lambda)$. The standard notation for the net will be $\left(a_{\lambda}\right)_{\lambda \in \Lambda}$.

Let $S$ be a ring. Let $<$ be the relation on $S$ defined by $s<t$ if $t s=s$ for $s, t \in S$.

Proposition 2.2. Let $S$ be a ring with the relation just defined.

(i) The relation $<$ is transitive.

(ii) If $s, t \in S$ and $t$ is idempotent, then $s<t$ if and only if $s \subseteq t S$.

(iii) For every $s, t \in S, s<t$ and $t<s$ if and only if $s$ and $t$ are both idempotent and $s=t S$. In particular, for every $s \in S, s<s$ if and only if $s$ is idempotent.

(iv) For every $s, t \in S, s<t$ in $S$ if and only if $1-t<1-s$ in the opposite ring $S^{\text {op }}$ of $S$. 
Proof. Properties (i), (ii) and (iv) are trivial. For (iii), suppose that $s<t$ and $t<s$. Then $t s=s$ and $s t=t$, so that $s^{2}=s(t s)=t s=s$. By symmetry, $t$ also is idempotent. Now (iii) follows from (ii).

Let $\left(s_{\lambda}\right)_{\lambda \in \Lambda}$ be a net in a ring $S$ with the transitive relation $<$ just defined. Then:

(1) From $s_{\mu} s_{\lambda}=s_{\lambda}$ it follows that $s_{\lambda} S \subseteq s_{\mu} S$ whenever $\lambda \leq \mu$, so that $\left(s_{\lambda} S\right)_{\lambda \in \Lambda}$ is a net in the set $\mathscr{L}\left(S_{S}\right)$ with the transitive relation $\subseteq$.

(2) The canonical projections $S / s_{\lambda} S \rightarrow S / s_{\mu} S$ give a direct system of right $S$ modules over the upward directed set $\Lambda$. We shall denote the direct limit $S / \bigcup_{\lambda \in \Lambda} s_{\lambda} S$ of this direct system by $\overline{\lim }_{S}\left(s_{\lambda}\right)_{\lambda \in \Lambda}$, and call it the upper limit of the net $\left(s_{\lambda}\right)_{\lambda \in \Lambda}$.

(3) From Proposition 2.2(iv) it follows that $\left(1-s_{\lambda}\right)_{\lambda \in \Lambda^{\text {op }}}$ is a net in $S^{\text {op }}$ defined on the opposite partially ordered set $\Lambda^{\text {op }}$ of $\Lambda$. Thus in the ring $S$ we have that $S\left(1-s_{\mu}\right) \subseteq S\left(1-s_{\lambda}\right)$ for $\lambda \leq \mu$ in $\Lambda$, so that the canonical projections $S / S\left(1-s_{\mu}\right) \rightarrow S / S\left(1-s_{\lambda}\right)$ give a direct system of left $S$-modules over $\Lambda^{\text {op }}$ ( $\Lambda^{\mathrm{op}}$ is upward directed because $\Lambda$ is downward directed). The direct limit of this direct system of left $S$-modules is $S / \bigcup_{\lambda \in \Lambda} S\left(1-s_{\lambda}\right)$. We shall denote it by $\underline{\lim }_{S}\left(s_{\lambda}\right)_{\lambda \in \Lambda}$, and call it the lower limit of the net $\left(s_{\lambda}\right)_{\lambda \in \Lambda}$. It coincides with the upper limit of the net $\left(1-s_{\lambda}\right)_{\lambda \in \Lambda^{\mathrm{op}}}$, which is a right $S^{\mathrm{op}}$-module.

Proposition 2.3. Let $\left(s_{\lambda}\right)_{\lambda \in \Lambda}$ be a net in a ring $S$. Then:

(i) The upper limit $\varlimsup_{S}\left(s_{\lambda}\right)_{\lambda \in \Lambda}$ is a cyclic flat right $S$-module.

(ii) The exact sequence

$$
0 \rightarrow \bigcup_{\lambda \in \Lambda} s_{\lambda} S \rightarrow S \rightarrow \varlimsup_{\lim _{S}}\left(s_{\lambda}\right)_{\lambda \in \Lambda} \rightarrow 0
$$

is pure, and $\bigcup_{\lambda \in \Lambda} s_{\lambda} S$ is a flat right ideal of $S$.

(iii) The upper limit $\varlimsup_{S}\left(s_{\lambda}\right)_{\lambda \in \Lambda}$ is projective if and only if there exists $\lambda_{0} \in \Lambda$ such that $s_{\lambda_{0}} S=s_{\lambda} S$ for any $\lambda \in \Lambda, \lambda \geq \lambda_{0}$. In this case, $s_{\lambda}^{2}=s_{\lambda}$ for any $\lambda \in \Lambda$, $\lambda>\lambda_{0}$.

(iv) The lower limit $\underline{\lim }_{S}\left(s_{\lambda}\right)_{\lambda \in \Lambda}$ is a cyclic flat left $S$-module.

(v) The exact sequence

$$
0 \rightarrow \bigcup_{\lambda \in \Lambda} S\left(1-s_{\lambda}\right) \rightarrow S \rightarrow \underline{\lim }_{S}\left(s_{\lambda}\right)_{\lambda \in \Lambda} \rightarrow 0
$$

is pure, and $\bigcup_{\lambda \in \Lambda} S\left(1-s_{\lambda}\right)$ is a flat left ideal of $S$.

(vi) The lower limit $\underline{\lim }_{S}\left(s_{\lambda}\right)_{\lambda \in \Lambda}$ is projective if and only if there exists $\mu_{0} \in \Lambda$ such that $S\left(1-s_{\mu_{0}}\right)=S\left(1-s_{\lambda}\right)$ for any $\lambda \in \Lambda, \lambda \leq \mu_{0}$. In this case, $s_{\lambda}^{2}=s_{\lambda}$ for any $\lambda \in \Lambda, \lambda<\mu_{0}$. 
Proof. In order to show that $\varlimsup_{S}\left(s_{\lambda}\right)_{\lambda \in \Lambda}=S / \bigcup_{\lambda \in \Lambda} s_{\lambda} S$ is flat, it is enough to prove that $\left(\bigcup_{\lambda \in \Lambda} s_{\lambda} S\right) L=\left(\bigcup_{\lambda \in \Lambda} s_{\lambda} S\right) \cap L$ for any left ideal $L$ [Anderson and Fuller 1992, Lemma 19.18]. The inclusion $\left(\bigcup_{\lambda \in \Lambda} s_{\lambda} S\right) L \subseteq\left(\bigcup_{\lambda \in \Lambda} s_{\lambda} S\right) \cap L$ always holds. If $x \in\left(\bigcup_{\lambda \in \Lambda} s_{\lambda} S\right) \cap L$, then $x=s_{\mu} y$ for suitable $\mu \in \Lambda$ and $y \in S$. As $\Lambda$ does not have a greatest element, there exists $v>\mu$, so that $x=s_{\mu} y=s_{\nu} s_{\mu} y=$ $s_{\nu} x \in\left(\bigcup_{\lambda \in \Lambda} s_{\lambda} S\right) L$. This shows (i).

Statement (ii) follows from (i), because every short exact sequence that ends with a flat module is pure.

To prove (iii), assume that $\lambda_{0} \in \Lambda$ is such that $s_{\lambda_{0}} S=s_{\lambda} S$ for $\lambda \in \Lambda, \lambda \geq \lambda_{0}$. Then, for every $\lambda>\lambda_{0}$, there exists $a \in S$ such that $s_{\lambda}=s_{\lambda_{0}} a=s_{\lambda} s_{\lambda_{0}} a=s_{\lambda}^{2}$. Thus $\bigcup_{\lambda \in \Lambda} s_{\lambda} S$ is generated by an idempotent, hence it is a direct summand of $S$. Conversely, if $\varlimsup_{S}\left(s_{\lambda}\right)_{\lambda \in \Lambda}$ is projective, then $\bigcup_{\lambda \in \Lambda} s_{\lambda} S$ is principal, so that there is a $\lambda_{0}$ with $s_{\lambda_{0}} S=s_{\lambda} S$ for every $\lambda \geq \lambda_{0}$.

The proofs of statements (iv) to (vi) are similar.

Notice that every countable partially ordered set $\Lambda$ satisfying condition (1) of Definition 2.1 contains an upward and downward cofinal subset order-isomorphic to the ordered set $\mathbb{Z}$. Thus we can always suppose $\Lambda=\mathbb{Z}$ for every countably infinite net.

Examples 2.4. Let $S$ be a ring, and let $\Lambda$ be a partially ordered set satisfying condition (1) of Definition 2.1.

(1) Let $e \in S$ be an idempotent. Then the constant map $\Lambda \rightarrow S$ defined by $\lambda \mapsto e$ for every $\lambda \in \Lambda$ is a net whose upper limit is the projective right module $S / e S \cong(1-e) S$ and whose lower limit is the projective left module $S / S(1-e) \cong S e$.

(2) More generally, let $\varphi: \Lambda \rightarrow S$ be a net such that, for every $\lambda \in \Lambda, \varphi(\lambda)=$ $e_{\lambda}$ is an idempotent of $S$. Equivalently, $\left\{e_{\lambda}\right\}_{\lambda \in \Lambda}$ is a family of, not necessarily distinct, idempotents of $S$ such that $e_{\lambda} S \subseteq e_{\mu} S$ for any pair $\lambda<\mu$ in $\Lambda$. The upper limit of this net is $S / \bigcup_{\lambda \in \Lambda} e_{\lambda} S$ and the lower limit is $S / \bigcup_{\lambda \in \Lambda} S\left(1-e_{\lambda}\right)$. Moreover, the upper limit is projective if and only if the family $\left\{e_{\lambda} S\right\}_{\lambda \in \Lambda}$ has a greatest element, $e_{\lambda_{0}} S$ say, and in this case $\varlimsup_{S}\left(e_{\lambda}\right)_{\lambda \in \Lambda} \cong\left(1-e_{\lambda_{0}}\right) S$. Dually, the lower limit is projective if and only if $\left\{e_{\lambda} S\right\}_{\lambda \in \Lambda}$ has a least element, $e_{\lambda_{1}} S$ say, and then $\underline{\lim }_{S}\left(e_{\lambda}\right)_{\lambda \in \Lambda} \cong S e_{\lambda_{1}}$.

\section{Tensoring nets with bimodules}

Now we study how elements of nets act on bimodules producing interesting pure exact sequences.

Proposition 3.1. Let $R$ and $S$ be rings, let ${ }_{S} M_{R}$ be an $S$-R-bimodule, and let ${ }_{R} N_{S}$ be an $R$-S-bimodule. Assume $\left(s_{\lambda}\right)_{\lambda \in \Lambda}$ is a net in the ring $S$. Then: 
(i) $\left(s_{\lambda} M\right)_{\lambda \in \Lambda}$ is a net in $\mathscr{L}\left(M_{R}\right)$ with the transitive relation $\subseteq$, and $\left(M / s_{\lambda} M\right)_{\lambda \in \Lambda}$ is a directed system of right $R$-modules.

(ii) There is an exact sequence

$$
0 \rightarrow\left(\bigcup_{\lambda \in \Lambda} s_{\lambda} S\right) \otimes_{S} M \rightarrow S \otimes_{S} M \rightarrow\left(\overline{\lim }_{S}\left(s_{\lambda}\right)_{\lambda \in \Lambda}\right) \otimes_{S} M \rightarrow 0
$$

which is a pure sequence of right $R$-modules.

(iii) $\lim _{\longrightarrow} M / s_{\lambda} M \cong M / \sum_{\lambda \in \Lambda} s_{\lambda} M \cong \varlimsup_{S}\left(s_{\lambda}\right)_{\lambda \in \Lambda} \otimes_{S} M$.

(iv) The module $M_{R}$ is flat if and only if both $M / \sum_{\lambda \in \Lambda} s_{\lambda} M$ and $\sum_{\lambda \in \Lambda} s_{\lambda} M$ are flat.

(v) $\left(N\left(1-s_{\lambda}\right)\right)_{\lambda \in \Lambda}$ is a net in $\mathscr{L}\left({ }_{R} N\right)$ with the transitive relation $\subseteq$, and

$$
\left(N / N\left(1-s_{\lambda}\right)\right)_{\lambda \in \Lambda}
$$

is a directed system of left $R$-modules.

(vi) There is an exact sequence

$0 \rightarrow N \otimes_{S}\left(\bigcup_{\lambda \in \Lambda} S\left(1-s_{\lambda}\right)\right) \rightarrow N \otimes_{S} S \rightarrow N \otimes_{S}\left(\underline{\lim }_{S}\left(s_{\lambda}\right)_{\lambda \in \Lambda}\right) \rightarrow 0$

which is a pure sequence of left $R$-modules.

(vii) $\lim _{\longrightarrow} N / N\left(1-s_{\lambda}\right) \cong N / \sum_{\lambda \in \Lambda} N\left(1-s_{\lambda}\right) \cong N \otimes_{S} \underline{\lim }_{S}\left(s_{\lambda}\right)_{\lambda \in \Lambda}$.

(viii) The left module ${ }_{R} N$ is flat if and only if both $N / \sum_{\lambda \in \Lambda} N\left(1-s_{\lambda}\right)$ and $\sum_{\lambda \in \Lambda} N\left(1-s_{\lambda}\right)$ are flat.

Proof. (i) follows easily from the fact that $\left(s_{\lambda}\right)_{\lambda \in \Lambda}$ is a net in $S$. (ii) follows from Proposition 2.3(ii) and the associativity of tensor product.

Let $\left\{e_{\lambda}\right\}_{\lambda \in \Lambda}$ be the canonical basis of the free right $S$-module $S^{(\Lambda)}$. Setting $f\left(e_{\lambda}\right)=s_{\lambda}$ we obtain from Proposition 2.3(ii) an exact sequence

$$
S^{(\Lambda)} \stackrel{f}{\rightarrow} S \rightarrow \overline{\lim }_{S}\left(s_{\lambda}\right)_{\lambda \in \Lambda} \rightarrow 0 .
$$

Tensoring this exact sequence with $M$, we get that $\overline{\lim }_{S}\left(s_{\lambda}\right)_{\lambda \in \Lambda} \otimes_{S} M$ is isomorphic to the cokernel of $f \otimes_{S} 1_{M}: S^{(\Lambda)} \otimes_{S} M \rightarrow S \otimes_{S} M$. Thus (iii) follows from (ii).

To prove (iv) notice that the sequence

$$
0 \rightarrow \bigcup_{\lambda \in \Lambda} s_{\lambda} S \otimes_{S} M \cong \sum_{\lambda \in \Lambda} s_{\lambda} M \rightarrow S \otimes_{S} M \cong M \rightarrow \varlimsup_{\lim _{S}}\left(s_{\lambda}\right)_{\lambda \in \Lambda} \otimes_{S} M \rightarrow 0
$$

is pure.

The proof of statements (v) to (viii) is similar.

In the next examples we apply Proposition 3.1 to Examples 2.4(2). 
Examples 3.2. Let $M_{R}$ be an arbitrary right module over a ring $R$. Let $S$ be the endomorphism ring $\operatorname{End}\left(M_{R}\right)$, so that ${ }_{S} M_{R}$ is a bimodule.

(1) As in Examples 2.4(2), let $\left(e_{\lambda}\right)_{\lambda \in \Lambda}$ be a net of idempotents of $S$. Then, for each $\lambda \in \Lambda, e_{\lambda} M$ is a direct summand of $M$ and $e_{\lambda} S_{S} \cong \operatorname{Hom}_{R}\left(M, e_{\lambda} M\right)_{S}$. In view of Proposition 2.3, $K=\bigcup_{\lambda \in \Lambda} \operatorname{Hom}_{R}\left(M, e_{\lambda} M\right)_{S}$ is a pure flat right ideal of $S$ and $S / K$ is a cyclic flat $S$-module. By Proposition 3.1, we obtain a pure exact sequence of right $R$-modules

$$
0 \rightarrow \sum_{\lambda \in \Lambda} e_{\lambda} M \rightarrow M \rightarrow M / \sum_{\lambda \in \Lambda} e_{\lambda} M \rightarrow 0
$$

(2) Nets as in (1) can be also constructed directly from a suitable family of direct summands of $M$. Let $\Lambda^{\prime}$ be a nonempty, upward directed and downward directed subset of $\mathscr{L}\left(M_{R}\right)$ whose elements are direct summands of $M_{R}$. Let $\Lambda=\Lambda^{\prime} \times \mathbb{Z}$ be partially ordered with the lexicographic order, so that $\Lambda$ is upward directed and downward directed and does not have a greatest element and a least element. For every $\lambda \in \Lambda^{\prime}$ fix an idempotent $e_{\lambda} \in S$ with image $\lambda$. Let $\varphi: \Lambda \rightarrow S$ be defined by $\varphi:(\lambda, n) \mapsto e_{\lambda}$ for every $(\lambda, n) \in \Lambda$. Then $\left(e_{\lambda}\right)_{\lambda \in \Lambda}$ is a net of idempotents of $S$.

(3) Assume $M=\bigoplus_{\alpha \in A} M_{\alpha}$. Let $\Lambda$ be the set of all finite subsets of $A$. For each subset $\lambda$ of $A$, let $M_{\lambda}=\bigoplus_{\alpha \in \lambda} M_{\alpha}$, and let $e_{\lambda}$ be the idempotent endomorphism of $M$ with image $M_{\lambda}$ and kernel $M_{A \backslash \lambda}$. Then

$$
K=\sum_{\lambda \in \Lambda} e_{\lambda} S=\sum_{\lambda \in \Lambda} \operatorname{Hom}_{R}\left(M, e_{\lambda} M\right)=\bigoplus_{\alpha \in A} e_{\{\alpha\}} S
$$

is a pure and projective right ideal of $S$. Note that, if $M_{\alpha}$ is nonzero for every $\alpha \in A$, then $K=S$ if and only if $A$ is finite, but in any case $S / K \otimes_{S} M \cong M / \bigoplus_{\alpha \in A} M_{\alpha}=0$. The set $\Lambda$ has a least element $\varnothing$, and, when $A$ is finite, a greatest element $A$. However, taking $\Lambda^{\prime}=\Lambda \times \mathbb{Z}$ with the lexicographic order, we obtain a partially ordered set with the properties required for index sets of nets.

\section{All flat right modules and all flat left modules arise from suitable nets}

Let $I$ be a nonempty set, and let $R$ be a ring. Let $F_{R}=\{f: I \times\{1\} \rightarrow R \mid f((i, 1))=$ 0 for almost all $i \in I\}$. Then $F_{R}$ is a free right $R$-module isomorphic to $R_{R}^{(I)}$, and we will rather think of it as the right $R$-module of all columns indexed by $I$, with entries in $R$, and at most finitely many nonzero entries. Let $\left\{e_{i} \mid i \in I\right\}$ be the canonical basis of $F_{R}$.

Let $F^{0}=\{f:\{1\} \times I \rightarrow R \mid f((1, i))=0$ for almost all $i \in I\}$. Then $F^{0}$ is a free left $R$-module isomorphic to ${ }_{R} R^{(I)}$, and we will think of it as the set of all rows indexed by $I$, with entries in $R$, and at most finitely many nonzero entries. Also denote by $\left\{e_{i} \mid i \in I\right\}$ the canonical basis of ${ }_{R} F^{0}$. 
Let $\mathbb{R C F}(I, R)$ denote the ring of all square matrices indexed by $I \times I$ with only a finite number of nonzero entries in each row and each column. Then $\mathbb{R C E}(I,-)$ is a functor of the category of associative rings with identity into itself. Let $B(I, R)$ be the set of all square matrices indexed by $I \times I$, with entries from $R$, with at most finitely many nonzero entries. The set $B(I, R)$ is a two-sided ideal in $\mathbb{R} \mathbb{C} F(I, R)$. If $I$ is finite of cardinality $n, \mathbb{R C F}(I, R)=B(I, R)$ is the ring of all $n \times n$ square matrices over $R$.

Let $S(I,-)$ be a subfunctor of $\mathbb{R} \mathbb{C F}(I,-)$ with the following property: for every ring $R$, the subring $S(I, R)$ of $\mathbb{R} \mathbb{C F}(I, R)$ contains $B(I, R)$ (and contains the identity of $\mathbb{R C E}(I, R))$. For instance, $S(I, R)$ could be the ring $\mathbb{R C F}(I, R)$ itself; or the subring $B(I, R)+1_{\mathbb{R C F}(I, R)} \cdot R$, where $1_{\mathbb{R C F}(I, R)} \cdot R$ is the set of all scalar matrices; or $S(I, R)=B(I, R)+1_{\mathbb{R C F}(I, R)} \cdot \mathbb{Z}$.

From now on, in this section, we specialize nets to the rings $S=S(I, R)$. Notice that $F$ is an $S$ - $R$-bimodule and $F^{0}$ is an $R$ - $S$-bimodule.

Let $\left(A_{\lambda}\right)_{\lambda \in \Lambda}$ be a net in $S$. We can apply Proposition 3.1 and obtain a flat right $R$-module $\varlimsup_{S}\left(A_{\lambda}\right)_{\lambda \in \Lambda} \otimes_{S} F \cong F_{R} / \bigcup_{\lambda \in \Lambda} A_{\lambda} F_{R}$ with presentation

$$
0 \rightarrow \bigcup_{\lambda \in \Lambda} A_{\lambda} S \otimes_{S} F \cong \sum_{\lambda \in \Lambda} A_{\lambda} F \rightarrow S \otimes_{S} F \cong F \rightarrow \varlimsup_{\lim _{S}}\left(A_{\lambda}\right)_{\lambda \in \Lambda} \otimes_{S} F \rightarrow 0
$$

and a flat left $R$-module $F^{0} \otimes_{S} \underline{\lim }_{S}\left(A_{\lambda}\right)_{\lambda \in \Lambda} \cong F^{0} / \bigcup_{\lambda \in \Lambda} F^{0}\left(1-A_{\lambda}\right)$ with presentation

$$
\begin{aligned}
& 0 \rightarrow F^{0} \otimes_{S} \bigcup_{\lambda \in \Lambda} S\left(1-A_{\lambda}\right) \cong \sum_{\lambda \in \Lambda} F^{0}\left(1-A_{\lambda}\right) \rightarrow F^{0} \otimes_{S} S \cong F^{0} \rightarrow \\
& \rightarrow F^{0} \otimes_{S} \underline{\lim }_{S}\left(A_{\lambda}\right)_{\lambda \in \Lambda} \rightarrow 0 .
\end{aligned}
$$

In the following theorem we show that all flat right $R$-modules and all flat left $R$-modules arise in this way from a net in $S=S(I, R)$ for a suitable set $I$.

Theorem 4.1. Let $F_{R} \rightarrow M_{R}$ be an epimorphism of the free right $R$-module $F_{R} \cong$ $R_{R}^{(I)}$ onto a flat right $R$-module $M_{R}$. Then there exists a net $\left(A_{\lambda}\right)_{\lambda \in \Lambda}$ in $S=S(I, R)$ with $A_{\lambda} \in B(I, R)$ for every $\lambda \in \Lambda$ and $\overline{\lim }_{S}\left(A_{\lambda}\right)_{\lambda \in \Lambda} \otimes_{S} F \cong M_{R}$. Dually, let ${ }_{R} F^{0} \rightarrow{ }_{R} N$ be an epimorphism of the free left $R$-module ${ }_{R} F^{0} \cong{ }_{R} R^{(I)}$ onto a flat left $R$-module ${ }_{R} N$. Then there exists a net $\left(B_{\lambda}\right)_{\lambda \in \Lambda}$ in $S=S(I, R)$ with $1-B_{\lambda} \in$ $B(I, R)$ for every $\lambda \in \Lambda$ and $F^{0} \otimes_{S} \underline{\lim }_{S}\left(B_{\lambda}\right)_{\lambda \in \Lambda} \cong{ }_{R} N$.

Proof. For the proof, we need the following result, which is a corollary of a theorem due to O. Villamayor [Lam 1999, Theorem 4.23].

Proposition 4.2. Let $\psi: F_{R} \rightarrow M_{R}$ be an epimorphism of the free right $R$-module $F_{R} \cong R_{R}^{(I)}$ onto a flat right $R$-module $M_{R}$. Then for any finitely generated submodule $C$ of $\operatorname{ker} \psi$ there exists $A \in B(I, R)$ such that $\psi\left(A F_{R}\right)=0$ and $A x=x$ for every $x \in C$. 
Proof. The proof of [Lam 1999, Theorem 4.23(1) $\Rightarrow(2)]$ shows that for any $c \in$ ker $\psi$ there exists $\vartheta \in \operatorname{Hom}(F, \operatorname{ker} \psi)$ with $\vartheta(c)=c$ such that $\vartheta\left(e_{i}\right)=0$ for almost all $i \in I$. The proof by induction of [Lam 1999, Theorem 4.23, (2) $\Rightarrow$ (3)] shows that for any $c_{1}, \ldots, c_{n} \in \operatorname{ker} \psi$ there exists $\vartheta \in \operatorname{Hom}(F, \operatorname{ker} \psi)$ with $\vartheta\left(c_{j}\right)=c_{j}$ for all $j=1, \ldots, n$ and such that $\vartheta\left(e_{i}\right)=0$ for almost all $i \in I$. If $c_{1}, \ldots, c_{n}$ generate the submodule $C$ of $\operatorname{ker} \psi$, then the matrix $A$ associated to $\vartheta$ with respect to the basis $\left\{e_{i} \mid i \in I\right\}$ has the required properties.

We are now ready for the proof of Theorem 4.1. Let $\psi: F_{R} \rightarrow M_{R}$ be an epimorphism of $F_{R}$ onto a flat right $R$-module $M_{R}$, and let $K$ be the kernel of $\psi$.

Suppose that $K$ is not finitely generated. Let $G$ be a set of generators of $K$. Let $\mathscr{P}_{\text {fin }}(G)$ denote the set of all finite subsets of $G$, partially ordered by set inclusion. Let $\mathbb{Z}^{-}$be the set of negative integers with its usual order, and let $\Lambda$ be the disjoint union of $\mathbb{Z}^{-}$and $\mathscr{P}_{\text {fin }}(G)$. Define $z<H$ for every $z \in \mathbb{Z}^{-}$and every $H \in \mathscr{P}_{\text {fin }}(G)$, so that $\Lambda$ turns out to be upward directed and downward directed, without a greatest element and without a least element. In order to define a net $\left\{A_{\lambda} \mid \lambda \in \Lambda\right\}$ in $S$, first of all set $A_{z}=0$ for $z \in \mathbb{Z}^{-}$. Then define, for each $H \in \mathscr{P}_{\text {fin }}(G)$, a matrix $A_{H} \in B(I, R)$ by induction on the cardinality $|H|$ of $H$. For $H=\varnothing$, set $A_{\varnothing}=0$. Let $H \in \mathscr{P}_{\text {fin }}(G)$ with $|H|>0$ and suppose that $A_{H^{\prime}}$ has already been defined for every $H^{\prime} \in \mathscr{P}_{\text {fin }}(G)$ with $\left|H^{\prime}\right|<|H|$. Since $H$ has only finitely many proper subsets, the submodule $C$ of $K$ generated by $H$ and by all $A_{H^{\prime}} F_{R}$ when $H^{\prime}$ ranges in the set of all proper subsets of $H$ is a finitely generated submodule of $K$. By Proposition 4.2, there exists $A_{H} \in B(I, R)$ such that $A_{H} x=x$ for every $x \in C$ and $A_{H} F_{R} \subseteq K$. This completes the definition of the matrix $A_{H}$. Notice that $A_{H^{\prime}} F_{R} \subseteq C$, so that $A_{H} A_{H^{\prime}}=A_{H^{\prime}}$ whenever $H^{\prime} \subset H$. Thus $\left(A_{\lambda}\right)_{\lambda \in \Lambda}$ is a net with the required properties.

If the module $K$ is finitely generated, there is a finite subset $J$ of $I$ with $K \subseteq$ $\bigoplus_{i \in J} e_{i} R$. Now $M_{R}$ is flat and

$$
M_{R} \cong F_{R} / K \cong\left(\bigoplus_{i \in J} e_{i} R / K\right) \oplus\left(\bigoplus_{i \in I \backslash J} e_{i} R\right) .
$$

Thus $\left(\bigoplus_{i \in J} e_{i} R\right) / K$ is flat and finitely presented, hence projective. It follows that $K$ is a direct summand of $\bigoplus_{i \in J} e_{i} R$. Thus $K$ is a direct summand of $F_{R}$ and there is an idempotent endomorphism $\varepsilon$ of $F_{R}$ with image $K$. Let $A$ be the matrix associated to $\varepsilon$ with respect to the basis $\left\{e_{i} \mid i \in I\right\}$ of $F_{R}$. Then the partially ordered set $\mathbb{Z}$ of the integers with the matrices $A_{z}=A$ for every $z \in \mathbb{Z}$ form a net in $S$ with upper limit $\varlimsup_{\lim _{S}}\left(A_{z}\right)_{z \in \mathbb{Z}} \otimes_{S} F \cong F / \sum_{z \in \mathbb{Z}} A_{z} F \cong M_{R}$; cf. Examples 2.4(1). This concludes the case of $K$ finitely generated.

Dually, let ${ }_{R} F^{0} \rightarrow{ }_{R} N$ be an epimorphism of ${ }_{R} F^{0}$ onto a flat left $R$-module ${ }_{R} N$. Passing to the opposite ring $R^{\text {op }}$ of $R$, one has an epimorphism $F \rightarrow N$ of 
the free right $R^{\mathrm{op}}$-module $F$ onto the flat right $R^{\mathrm{op}}$-module $N$. By applying to this epimorphism the first part of the statement, which we have just proved, we see that there exists a net $\left(C_{\lambda}\right)_{\lambda \in \Lambda}$ in $S\left(I, R^{\text {op }}\right)$ with $\overline{\lim }\left(C_{\lambda}\right)_{\lambda \in \Lambda} \otimes F \cong F / \sum_{\lambda \in \Lambda} C_{\lambda} F \cong N$ as right $R^{\mathrm{op}}$-modules. In particular, the $C_{\lambda}$ 's belong to $S\left(I, R^{\mathrm{op}}\right)$, and $C_{\mu} C_{\lambda}=C_{\lambda}$ whenever $\lambda, \mu \in \Lambda$ and $\lambda<\mu$. Viewing these objects as left $R$-modules again and remarking that transposition is an isomorphism $\operatorname{tr}: S\left(I, R^{\mathrm{op}}\right) \rightarrow(S(I, R))^{\mathrm{op}}$, we have that the $C_{\lambda}^{\mathrm{tr}}$ 's belong to $S(I, R)$, that $C_{\lambda}^{\mathrm{tr}} C_{\mu}^{\mathrm{tr}}=C_{\lambda}^{\mathrm{tr}}$ in $S(I, R)$ whenever $\lambda<\mu$, and ${ }_{R} N \cong{ }_{R} F^{0} / \sum_{\lambda \in \Lambda}\left({ }_{R} F^{0}\right) C_{\lambda}^{\mathrm{tr}}$. From $C_{\lambda}^{\mathrm{tr}} C_{\mu}^{\mathrm{tr}}=C_{\lambda}^{\mathrm{tr}}$ for $\lambda<\mu$, we obtain that $\left(1-C_{\lambda}^{\mathrm{tr}}\right)\left(1-C_{\mu}^{\mathrm{tr}}\right)=1-C_{\mu}^{\mathrm{tr}}$ in $S=S(I, R)$ for $\lambda<\mu$. Denoting the set $\Lambda$ with the inverse order by $\Lambda^{\mathrm{op}}$, we see that there is a net $\left(1-C_{\lambda}^{\mathrm{tr}}\right)_{\lambda \in \Lambda^{\mathrm{op}}}$ in $S$ and

$$
F^{0} \otimes_{S} \underline{\lim }_{S}\left(1-C_{\lambda}^{\mathrm{tr}}\right)_{\lambda \in \Lambda^{\mathrm{op}}} \cong{ }_{R} F^{0} / \sum_{\lambda \in \Lambda}{ }_{R} F^{0} C_{\lambda}^{\mathrm{tr}} \cong{ }_{R} N
$$

Remark 4.3. Let $S$ and $S^{\prime}$ be rings. A ring homomorphism $f: S \rightarrow S^{\prime}$ induces for every net $\left(s_{\lambda}\right)_{\lambda \in \Lambda}$ in $S$ a net $\left(f\left(s_{\lambda}\right)\right)_{\lambda \in \Lambda}$ in $S^{\prime}$.

For instance, a ring homomorphism $g: R \rightarrow R^{\prime}$ induces a ring homomorphism $\tilde{g}=S(I, g): S=S(I, R) \rightarrow S^{\prime}=S\left(I, R^{\prime}\right)$. If $\left(A_{\lambda}\right)_{\lambda \in \Lambda}$ is a net in $S$, then

$$
\left(\varlimsup_{S}\left(A_{\lambda}\right)_{\lambda \in \Lambda} \otimes_{S} F_{R}\right) \otimes_{R} R^{\prime} \cong \varlimsup_{S^{\prime}}\left(\tilde{g}\left(A_{\lambda}\right)\right)_{\lambda \in \Lambda} \otimes_{S^{\prime}} F_{R^{\prime}}^{\prime}
$$

and

$$
R^{\prime} \otimes_{R}\left({ }_{R} F^{0} \otimes_{S} \underline{\lim }_{S}\left(A_{\lambda}\right)_{\lambda \in \Lambda}\right) \cong{ }_{R^{\prime}}\left(F^{\prime}\right)^{0} \otimes_{S^{\prime}} \underline{\lim }_{S^{\prime}}\left(\tilde{g}\left(A_{\lambda}\right)\right)_{\lambda \in \Lambda},
$$

where we have denoted by $F_{R^{\prime}}^{\prime}$ the free right $R^{\prime}$-module of rank $|I|$ and by $R^{\prime}\left(F^{\prime}\right)^{0}$ the free left $R^{\prime}$-module of same rank.

We will be particularly interested in the case in which $g: R \rightarrow R / J(R)$ is the canonical projection.

\section{Two examples of flat modules}

As a first example, we shall consider the flat module $F_{R} / G$ introduced in the seminal paper [Bass 1960]. Fix a sequence $a_{n}(n \geq 1)$ of elements of a given ring $R$, let $F_{R}$ be the free right $R$-module with basis $\left\{e_{n} \mid n \geq 1\right\}$, and let $G$ be the submodule of $F_{R}$ generated by the elements $y_{n}=e_{n}-e_{n+1} a_{n}$, for $n \geq 1$. It is known that $G$ is a free $R$-module with basis $\left\{y_{n} \mid n \geq 1\right\}$ and $F_{R} / G$ is a flat module [Bass 1960; Anderson and Fuller 1992, Lemma 28.1]. The module $F_{R} / G$ is projective, that is, $G$ is a direct summand of $F_{R}$, if and only if all the descending chains $R a_{n} \supseteq R a_{n+1} a_{n} \supseteq R a_{n+2} a_{n+1} a_{n} \supseteq \cdots$, for $n \geq 1$, are stationary [Azumaya 1987, Theorem 26]. 
Let $S$ be the ring $S\left(\mathbb{Z}^{+}, R\right)$, where $\mathbb{Z}^{+}$denotes the set of all positive integers. Let $\left(A_{n}\right)_{n \in \mathbb{Z}}$ be the net in $S$ defined by $A_{n}=0$ for $n \leq 0$, and

$$
A_{n}=\left(\begin{array}{cccc|c}
1 & 0 & \cdots & 0 & \\
0 & 1 & \cdots & 0 & 0 \\
\vdots & \vdots & \ddots & \vdots & \\
0 & 0 & \cdots & 1 & \\
\hline-a_{n} \ldots a_{2} a_{1} & -a_{n} \ldots a_{2} & \cdots & -a_{n} & \\
& 0 & & & 0
\end{array}\right)
$$

for $n \geq 1$. An easy computation shows that $A_{n}^{2}=A_{n}$ for any $n \in \mathbb{Z}$. In particular, $A_{n} F_{R}$ is a direct summand of $F_{R}$.

Proposition 5.1. For every $n \geq 1$ the right $R$-module $A_{n} F_{R}$ is the free submodule of $G$ generated by $y_{1}, \ldots, y_{n}$.

Proof. We must show that the right $R$-module $A_{n} F_{R}$, generated by

$$
e_{1}-e_{n+1} a_{n} \ldots a_{2} a_{1}, \ldots, e_{n}-e_{n+1} a_{n}
$$

coincides with the right module generated by $y_{1}=e_{1}-e_{2} a_{1}, \ldots, y_{n}=e_{n}-e_{n+1} a_{n}$.

$$
\begin{aligned}
& e_{i}-e_{n+1} a_{n} \ldots a_{i+1} a_{i}=\left(e_{i}-e_{i+1} a_{i}\right)+\left(e_{i+1}-e_{i+2} a_{i+1}\right) a_{i} \\
& \quad+\left(e_{i+2}-e_{i+3} a_{i+2}\right) a_{i+1} a_{i}+\cdots+\left(e_{n}-e_{n+1} a_{n}\right) a_{n-1} a_{n-2} \ldots a_{i} .
\end{aligned}
$$

Conversely, for $i<n$, we see that $y_{i}=e_{i}-e_{i+1} a_{i}=\left(e_{i}-e_{n+1} a_{n} \ldots a_{i+1} a_{i}\right)-$ $\left(e_{i+1}-e_{n+1} a_{n} \ldots a_{i+1}\right) a_{i}$.

Thus $\sum_{n \in \mathbb{Z}} A_{n} F_{R}=G$ and $\varlimsup_{\lim _{S}}\left(A_{\lambda}\right)_{\lambda \in \Lambda} \otimes_{S} F \cong F_{R} / G$.

Let $E=\operatorname{End}_{R}\left(F_{R}\right)$. Let $K_{1}=\bigcup_{n=1}^{\infty} \operatorname{Hom}_{R}\left(F, \sum_{j=1}^{n} e_{j} R\right)$, and let $K_{2}=$ $\bigcup_{n=1}^{\infty} \operatorname{Hom}_{R}\left(F, A_{n} F\right)$. In view of Examples 3.2, $K_{1}$ and $K_{2}$ are pure right ideals of $E$. Note that they are also projective [Lazard 1969, Théorème 3.2].

Proposition 5.2. The cyclic right $E$-modules $E / K_{1}$ and $E / K_{2}$ are flat and nonisomorphic. If the elements of the sequence $a_{n}(n \geq 1)$ belong to $J(R)$, then $E / K_{1} \otimes_{E} E / J(E) \cong E / K_{2} \otimes_{E} E / J(E)$.

Proof. Applying the functor $-\otimes_{E} F$ to the pure exact sequence

$$
0 \rightarrow K_{1} \rightarrow E \rightarrow E / K_{1} \rightarrow 0
$$

it follows that $E / K_{1} \otimes_{E} F=0$ (Examples 3.2). While applying the functor $-\otimes_{E} F$ to the pure exact sequence

$$
0 \rightarrow K_{2} \rightarrow E \rightarrow E / K_{2} \rightarrow 0
$$


it follows that $K_{2} \otimes_{E} F \cong G$, hence $E / K_{2} \otimes_{E} F \cong F / G$.

If $a_{n}(n \geq 1)$ is a sequence of elements in $J(R)$ and $g: R \rightarrow R / J(R)$ denotes the canonical projection, then $\tilde{g}\left(K_{1}\right)=\tilde{g}\left(K_{2}\right)$, so

$$
E / K_{1} \otimes_{E} E / J(E) \cong E / K_{2} \otimes_{E} E / J(E)
$$

in view of Remark 4.3.

The isomorphism $f: F \rightarrow G$ defined by $f\left(e_{n}\right)=y_{n}$ for every $n \geq 1$ induces an isomorphism between the projective ideals $K_{1}$ and $K_{2}$.

In the next proposition we give an example that was our initial motivation to define nets. We construct a countable net whose upper limit is nontrivial if and only and only if its lower limit is nontrivial; that is, the net produces a nontrivial right flat module if and only if it produces a nontrivial left flat module. This idea will be further developed and applied in the proof of Theorem 7.1.

Proposition 5.3. Let $S$ be a ring. Let $s$ and $u$ be elements of $S$ such that $u$ is invertible and $s^{2}=u s$. For every $m \in \mathbb{Z}$, set $s_{m}=u^{-m}\left(u^{-1} s\right) u^{m}$. Let $I=\sum_{m \in \mathbb{Z}} s_{m} S$, and let $L=\sum_{m \in \mathbb{Z}} S\left(1-s_{m}\right)$. Then:

(i) $\left(s_{m}\right)_{m \in \mathbb{Z}}$ is a net.

(ii) The right ideal I and the left ideal $L$ are projective. The right $S$-module $S / I$ and the left $S$-module $S / L$ are flat.

(iii) There exists $m \in \mathbb{Z}$ such that $s_{m}^{2}=s_{m}$ if and only if $s u^{-1} s=s$, if and only if $s_{m}^{2}=s_{m}$ for all $m \in \mathbb{Z}$.

(iv) The right ideal I is finitely generated if and only if the left ideal $L$ is finitely generated, if and only if $s u^{-2} s=u^{-1} s$.

Proof. (i) Direct computation shows that $s_{m}=s_{n} s_{m}$ for $m<n$.

(ii) Note that $S / I=\varlimsup_{S}\left(s_{m}\right)_{m \in \mathbb{Z}}$ and $S / L=\underline{\lim }_{S}\left(s_{m}\right)_{m \in \mathbb{Z}}$. By Proposition 2.3, $S / I$ is a flat right module and $S / L$ is a flat left module. Since, by [Lazard 1969 , Théorème 3.2], countably presented flat modules have projective dimension $1, I$ and $L$ are projective.

To prove (iii), observe that $s_{m}^{2}=s_{m}$ if and only if

$$
u^{-m}\left(u^{-1} s\right) u^{m} \cdot u^{-m}\left(u^{-1} s\right) u^{m}=u^{-m}\left(u^{-1} s\right) u^{m},
$$

if and only if $s u^{-1} s=s$, as claimed. As this condition does not depend on $m$, if one $s_{m}$ is idempotent all must be idempotent.

(iv) First observe that the identity $s_{m} s_{m+1}=s_{m+1}$ holds for some $m$ if and only if $s u^{-2} s=u^{-1} s$. As this condition does not depend on $m$, this happens if and only if $s_{m} s_{m+1}=s_{m+1}$ for all $m$. 
Since always $s_{m} S \subseteq s_{m+1} S$ for every $m, s u^{-2} s=u^{-1} s$ implies that $s_{m} S=s_{m+1} S$ for all $m$. Hence $I$ is principal.

Conversely, if $I_{S}$ is finitely generated, then $S / I$ is flat and finitely presented, hence projective, so by Proposition 2.3(iii) there exists $m$ such that $s_{m}^{2}=s_{m}$ and $I=s_{m} S$. Then $s_{m} S=s_{m+1} S$ implies $s_{m} s_{m+1}=s_{m+1}$, hence $s u^{-2} s=u^{-1} s$.

Similar arguments show the statement for $L$.

The symmetry in the conclusions of Proposition 5.3 can be explained through the following lemma, which is an observation based on [Zöschinger 1981, Satz 1.2]. See also [Puninski 2004, Section 3].

Lemma 5.4. Let $S$ be a ring, and let $s \in S$. There exists a unit $u$ such that $s^{2}=u s$ if and only if there exists $t \in S$ such that $t s=0$ and $s+t$ is a unit. In this situation, there exists a unit $v \in S$ such that $t^{2}=t v$.

Proof. Assume there exists a unit $u$ such that $s^{2}=u s$. Then $t=u-s$ satisfies the required properties. Conversely, if there exists $t \in S$ such that $t s=0$ and $s+t$ is a unit, then taking $u=s+t$ we have that $u s=s^{2}$. Note that then also $t u=t^{2}$.

\section{Lifting projective modules modulo the Jacobson radical}

In this section and the next we apply the theory developed earlier to the lifting of finitely generated projective modules modulo the Jacobson radical.

For every right (left) $R$-module $M_{R}\left({ }_{R} N\right)$, let $M^{*}=\operatorname{Hom}_{R}\left(M_{R}, R_{R}\right)\left(N^{*}=\right.$ $\left.\operatorname{Hom}_{R}\left({ }_{R} N,{ }_{R} R\right)\right)$ denote the dual of the module $M_{R}\left({ }_{R} N\right)$, which is a left (right) $R$-module. This defines a duality, that is, a contravariant equivalence, between the full subcategory of finitely generated projective right $R$-modules and the full subcategory of finitely generated projective left $R$-modules.

Consider a direct sum decomposition $P \oplus Q=(R / J(R))^{n}$ of the free right $R / J(R)$-module $(R / J(R))^{n}$, so that $P$ and $Q$ are two projective right $R / J(R)$ modules. It is easy to see that there exists a finitely generated projective right $R$ module $M_{R}$ such that $M / M J(R) \cong P$ if and only if there exists a finitely generated projective right $R$-module $Q_{R}^{\prime}$ such that $Q^{\prime} / Q^{\prime} J(R) \cong Q$, if and only if there exists a finitely generated projective left $R$-module ${ }_{R} N$ such that $N / J(R) N \cong$ $\operatorname{Hom}_{R}(Q, R / J(R))$, if and only if there exists a finitely generated projective left $R$-module ${ }_{R} P^{\prime}$ such that $P^{\prime} / J(R) P^{\prime} \cong \operatorname{Hom}_{R}\left(P, R / J(R)\right.$ ). (To prove this, let $M_{R}$ be a finitely generated projective right $R$-module such that $P \cong M / M J(R)$. Then $M / M J(R) \oplus Q \cong(R / J(R))^{n}$. By [Anderson and Fuller 1992, Lemma 17.17] there exists a finitely generated projective right $R$-module $Q^{\prime}$ such that $Q^{\prime} / Q^{\prime} J(R) \cong Q$ and $M \oplus Q^{\prime} \cong R^{n}$. Take $N=\operatorname{Hom}_{R}\left(Q^{\prime}, R\right)$.)

In this section we consider the problem of lifting these projective $R / J(R)$ modules to projective $R$-modules, not necessarily finitely generated. We need the following result of Bergman [Jøndrup 1976, Lemma 2.2]. 
Proposition 6.1. Let $Q$ and $Q^{\prime}$ be projective right $R$-modules, and let $\varphi: Q^{\prime} \rightarrow Q$ be a homomorphism. If the mapping $\bar{\varphi}: Q^{\prime} / Q^{\prime} J(R) \rightarrow Q / Q J(R)$ induced by $\varphi$ is a pure monomorphism, then $\varphi$ is a pure monomorphism.

Proof. First choose an $R$-module $P^{\prime}$ such that $Q^{\prime} \oplus P^{\prime}$ is free, then an $R$-module $P$ such that $\left(Q \oplus P^{\prime}\right) \oplus P$ is free. Let $\varepsilon: P^{\prime} \rightarrow P^{\prime} \oplus P$ denote the embedding. Substituting $\varphi: Q^{\prime} \rightarrow Q$ with $\varphi \oplus \varepsilon: Q^{\prime} \oplus P^{\prime} \rightarrow Q \oplus P^{\prime} \oplus P$, we may suppose that $Q$ and $Q^{\prime}$ are free. In order to show that $\varphi$ is a pure monomorphism, fix a finitely generated free direct summand $N^{\prime}$ of $Q^{\prime}$. Let $N$ be a finitely generated free direct summand of $Q$ containing $\varphi\left(N^{\prime}\right)$. Let $f: N \rightarrow Q$ and $f^{\prime}: N^{\prime} \rightarrow$ $Q^{\prime}$ be the inclusions, and $g: Q \rightarrow N, g^{\prime}: Q^{\prime} \rightarrow N^{\prime}$ be homomorphisms such that $g f=1_{N}$ and $g^{\prime} f^{\prime}=1_{N^{\prime}}$. If $\left.\varphi\right|_{N^{\prime}}: N^{\prime} \rightarrow N$ denotes the restriction of $\varphi$ : $Q^{\prime} \rightarrow Q$, then $\left.f \varphi\right|_{N^{\prime}}=\varphi f^{\prime}$. If - denotes reduction modulo $J(R)$, then $\overline{f^{\prime}}$ is a pure monomorphism, so that $\bar{\varphi} \overline{f^{\prime}}$ is a pure monomorphism. From $\left.f \varphi\right|_{N^{\prime}}=$ $\varphi f^{\prime}$, it follows that $\overline{\left.\varphi\right|_{N^{\prime}}}$ is a pure monomorphism. Thus the cokernel of $\overline{\left.\varphi\right|_{N^{\prime}}}$ is a flat finitely presented module, that is, a projective finitely generated module. In particular, $\overline{\left.\varphi\right|_{N^{\prime}}}$ is a split monomorphism. Let $h: N \rightarrow N^{\prime}$ be a homomorphism such that $1_{N^{\prime} / N^{\prime} J(R)}=\overline{\left.h \varphi\right|_{N^{\prime}}}$. Then $\left.h \varphi\right|_{N^{\prime}}$ is an automorphism of $N^{\prime}$, so that $\left.\varphi\right|_{N^{\prime}}$ is a split monomorphism. In particular, $\varphi$ is injective, and $\varphi\left(N^{\prime}\right)$ is a direct summand of $Q$ for every finitely generated free direct summand $N^{\prime}$ of $Q$. As $\varphi\left(Q^{\prime}\right)$ is the directed union of all these direct summands $\varphi\left(N^{\prime}\right), \varphi\left(Q^{\prime}\right)$ is a pure submodule of $Q$.

Corollary 6.2. Let $R$ be a ring with the property that for every projective right $R / J(R)$-module $P$ there exists a projective right $R$-module $Q$ with $Q / Q J(R) \cong P$. For every flat right $R / J(R)$-module $M$ of projective dimension $p d_{R / J(R)}(M) \leq 1$ there exists a flat right $R$-module $N$ of projective dimension $p d_{R}(N) \leq 1$ with $N / N J(R) \cong M$. Moreover, if $M$ is finitely generated, then $N$ can also be chosen finitely generated.

Proof. Apply Proposition 6.1 to a presentation

$$
0 \longrightarrow Q^{\prime} / Q^{\prime} J(R) \stackrel{\bar{\varphi}}{\longrightarrow} Q / Q J(R) \longrightarrow M \longrightarrow 0
$$

of the $R / J(R)$-module $M$ with $Q$ and $Q^{\prime}$ projective $R$-modules.

The hypothesis of Corollary 6.2 applies to all rings $R$ for which every projective right $R / J(R)$-module is free, and to all exchange rings $R$.

Proposition 6.3. Let $R$ be a ring and $X$ a set. Let $P \oplus Q=(R / J(R))^{(X)}$ be a direct sum decomposition of the free right $R / J(R)$-module $(R / J(R))^{(X)}$ as a direct sum of two projective right $R / J(R)$-modules $P$ and $Q$, and let $\pi:(R / J(R))^{(X)} \rightarrow P$ be the projection with kernel $Q$. The following statements are equivalent: 
(i) There exist a flat right $R$-module $M_{R}$ of projective dimension at most 1 , an epimorphism $\psi: R^{(X)} \rightarrow M_{R}$ and an isomorphism $\alpha: M_{R} / M_{R} J(R) \rightarrow P$ such that $\alpha \circ(\psi \otimes R / J(R))=\pi$.

(ii) There exists a projective right $R$-module $Q_{R}^{\prime}$ such that $Q^{\prime} / Q^{\prime} J(R) \cong Q$.

Proof. (i) $\Rightarrow$ (ii) Let $M_{R}$, $\psi$ and $\alpha$ have the properties stated in (i). We will show that the projective module $Q^{\prime}=\operatorname{ker} \psi$ has the property required in (ii). From the exact sequence

$$
0 \longrightarrow Q^{\prime} \longrightarrow R^{(X)} \stackrel{\psi}{\longrightarrow} M_{R} \longrightarrow 0,
$$

we get the exact sequence

$$
0 \longrightarrow Q^{\prime} / Q^{\prime} J(R) \longrightarrow(R / J(R))^{(X)} \stackrel{\psi \otimes R / J(R)}{\longrightarrow} M_{R} / M_{R} J(R) \longrightarrow 0 .
$$

Thus $Q=\operatorname{ker} \pi=\operatorname{ker}(\alpha \circ(\psi \otimes R / J(R)))=\operatorname{ker}(\psi \otimes R / J(R)) \cong Q^{\prime} / Q^{\prime} J(R)$.

(ii) $\Rightarrow$ (i) Let $Q_{R}^{\prime}$ be a projective $R$-module such that $Q^{\prime} / Q^{\prime} J(R) \cong Q$. Let $\rho: Q^{\prime} \rightarrow Q$ be an epimorphism with kernel $Q^{\prime} J(R)$. Denote by

$$
\varepsilon: Q \rightarrow(R / J(R))^{(X)}
$$

the embedding, which is a split monomorphism. As $\varepsilon \rho: Q_{R}^{\prime} \rightarrow(R / J(R))^{(X)}$ factors through the canonical projection of $R^{(X)}$ onto $(R / J(R))^{(X)}$, there is a commutative diagram

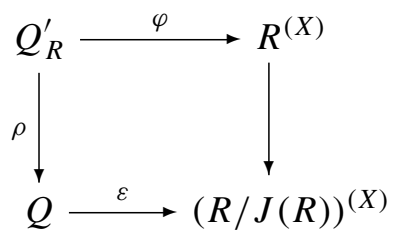

By Proposition 6.1 the mapping $\varphi$ is a pure monomorphism, so that its cokernel $M_{R}$ is a flat module of projective dimension $\leq 1$. Let $\psi: R^{(X)} \rightarrow M_{R}$ be the canonical projection. Applying $-\otimes_{R} R / J(R)$ to the pure exact sequence

$$
0 \longrightarrow Q_{R}^{\prime} \stackrel{\varphi}{\longrightarrow} R^{(X)} \stackrel{\psi}{\longrightarrow} M_{R} \rightarrow 0,
$$

we obtain an exact sequence that is the upper row of the commutative diagram

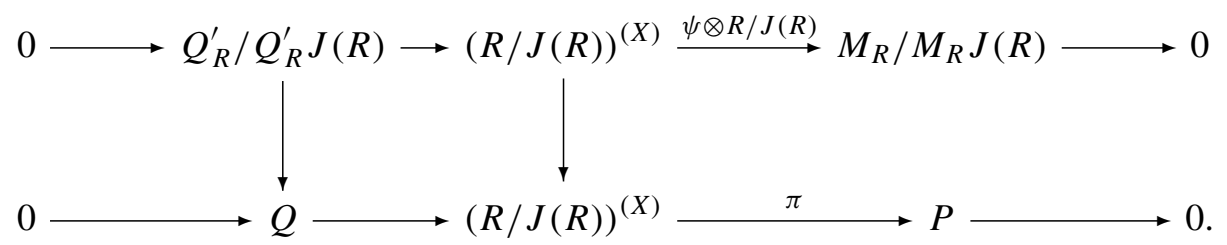

Here the two vertical arrows are isomorphisms (the vertical arrow on the right is the identity). Thus there is an isomorphism $\alpha: M_{R} / M_{R} J(R) \rightarrow P$ that completes the commutative diagram; that is, $\alpha \circ(\psi \otimes R / J(R))=\pi$. 


\section{Lifting finitely generated projective modules}

Theorem 7.1. Let $P \oplus Q=(R / J(R))^{n}$ be a direct sum decomposition of the finitely generated free right $R / J(R)$-module $(R / J(R))^{n}$ as a direct sum of two projective right $R / J(R)$-modules $P$ and $Q$. Then the following statements are equivalent:

(i) There exists a finitely generated flat right $R$-module $M_{R}$ such that $M / M J(R)$ is isomorphic to $P$.

(ii) There exists a finitely generated, countably presented, flat right $R$-module $M_{R}$ such that $M / M J(R) \cong P$.

(iii) There exists a projective right $R$-module $Q_{R}^{\prime}$ such that $Q^{\prime} / Q^{\prime} J(R) \cong Q$.

(iv) There exists a finitely generated flat left $R$-module ${ }_{R} N$ such that $N / J(R) N \cong$ $\operatorname{Hom}_{R}(Q, R / J(R))$.

(v) There exists a finitely generated, countably presented, flat left $R$-module ${ }_{R} N$ such that $N / J(R) N \cong \operatorname{Hom}_{R}(Q, R / J(R))$.

(vi) There exists a projective left $R$-module ${ }_{R} P^{\prime}$ such that $P^{\prime} / J(R) P^{\prime}$ is isomorphic to $\operatorname{Hom}_{R}(P, R / J(R))$.

Proof. Suppose that (i) holds. Let $M_{R}$ be a finitely generated flat right $R$-module such that $M / M J(R) \cong P$, where $P \oplus Q=(R / J(R))^{n}$. Let $\alpha: M / M J(R) \rightarrow P$ be an isomorphism, and let $\pi:(R / J(R))^{n} \rightarrow P$ be the projection with kernel $Q$. The onto mapping $\alpha^{-1} \pi:(R / J(R))^{n} \rightarrow M / M J(R)$ can be lifted to a homomorphism of right $R$-modules $\psi: R_{R}^{n} \rightarrow M_{R}$, which is necessarily onto by Nakayama's Lemma. Let $K=\operatorname{ker} \psi$ and consider the pure exact sequence of right $R$-modules

$$
0 \longrightarrow K \stackrel{\varepsilon}{\longrightarrow} R_{R}^{n} \stackrel{\psi}{\longrightarrow} M_{R} \longrightarrow 0 .
$$

Tensoring by $R / J(R)$, this induces the exact sequence

$$
0 \longrightarrow K / K J(R) \stackrel{\bar{\varepsilon}}{\longrightarrow}(R / J(R))^{n} \stackrel{\alpha^{-1} \pi}{\longrightarrow} M / M J(R) \longrightarrow 0,
$$

which splits because $M / M J(R) \cong P$ is projective. Moreover, $Q=\operatorname{ker} \pi=$ $\operatorname{ker}\left(\alpha^{-1} \pi\right) \cong K / K J(R)$. As (3) splits, there exists a left inverse

$$
\bar{\varphi}:(R / J(R))^{n} \rightarrow K / K J(R)
$$

of $\bar{\varepsilon}$ with kernel isomorphic to $M / M J(R) \cong P$. As $R^{n}$ is projective, $\bar{\varphi}$ can be lifted to a map $\varphi: R^{n} \rightarrow K$ making the diagram

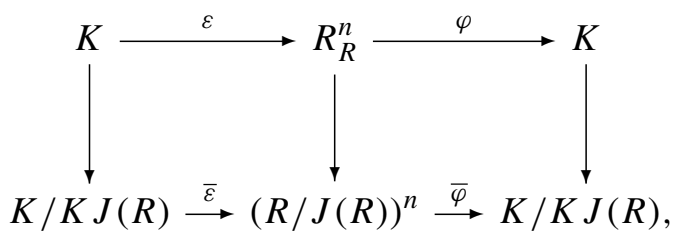


commute. In this diagram the vertical arrows are the natural projections. By Proposition 4.2, there exists $\omega: R^{n} \rightarrow K$ such that $\varepsilon \omega$ is the identity over $\varepsilon \varphi\left(R^{n}\right)$. Equivalently, $\omega \varepsilon \varphi=\varphi$. Denote by $\bar{\omega}:(R / J(R))^{n} \rightarrow K / K J(R)$ the induced homomorphism. Then $\overline{\omega \varepsilon \varphi}=\bar{\varphi}$. As $\overline{\varphi \varepsilon}$ is the identity mapping, $\overline{\omega-\varphi \varepsilon \omega}=\overline{\omega-\omega}=0$, so that $(\omega-\varphi \varepsilon \omega)\left(R^{n}\right) \subseteq K J(R)$, from which $\varepsilon(\omega-\varphi \varepsilon \omega)\left(R^{n}\right) \subseteq(J(R))^{n}$. Thus $\varepsilon(\omega-\varphi \varepsilon \omega) \in J\left(\operatorname{End}\left(R^{n}\right)\right)$ [Anderson and Fuller 1992, Corollary 17.12]. Set $\beta=1-\varepsilon(\omega-\varphi \varepsilon \omega)$, and note that $\beta$ is an invertible element of $\operatorname{End}\left(R^{n}\right)$. As $\omega \varepsilon \varphi=\varphi$, it is easy to see that $\beta \varepsilon \varphi=(\varepsilon \varphi)^{2}$. Observe that $\beta$ induces the identity endomorphism on $(R / J(R))^{n}$ and also that $\varepsilon \varphi$ induces the idempotent endomorphism $\overline{\varepsilon \varphi}$ on $(R / J(R))^{n}$, whose image is $K / K J(R) \cong Q$ and whose kernel is isomorphic to $P$. For any $m \in \mathbb{Z}$, let $A_{m}$ be the matrix associated to the endomorphism $\beta^{-m-1} \varepsilon \varphi \beta^{m}: R_{R}^{n} \rightarrow R_{R}^{n}$. By Proposition 5.3(i), $\left(A_{m}\right)_{m \in \mathbb{Z}}$ is a net in the ring $S=S(n, R)$ of $n \times n$ matrices over $R$. Hence the left $R$-module ${ }_{R} N=R^{n} / \bigcup_{m \in \mathbb{Z}} R^{n}\left(1-A_{m}\right)$ is flat. By Remark 4.3, if we apply isomorphism (2) with $R^{\prime}=R / J(R)$ and $g$ the canonical projection, and using that $\left(\tilde{g}\left(A_{m}\right)\right)_{m \in \mathbb{Z}}$ is the net in $S^{\prime}=S(n, R / J(R))$ constantly equal to the matrix $\bar{A}$ of the endomorphism $\overline{\varepsilon \varphi}$ of $(R / J(R))^{n}$, we see that

$$
(R / J(R))^{n} \otimes_{S^{\prime}} \underline{\lim }_{S^{\prime}}\left(\tilde{g}\left(A_{m}\right)\right)_{m \in \mathbb{Z}} \cong(R / J(R))^{n} \otimes_{S^{\prime}} \underline{\lim }_{S^{\prime}}(\bar{A})_{m \in \mathbb{Z}} .
$$

By Examples 2.4(1), $\underline{\lim }_{S^{\prime}}(\bar{A})_{m \in \mathbb{Z}} \cong S^{\prime} \bar{A}$. Thus

$$
\begin{aligned}
& N / J(R) N \cong R / J(R) \otimes_{R} N \cong(R / J(R))^{n} \otimes_{S^{\prime}} \underline{\lim }_{S^{\prime}}\left(\tilde{g}\left(A_{m}\right)\right)_{m \in \mathbb{Z}} \cong \\
& \cong(R / J(R))^{n} \otimes_{S^{\prime}} \underline{\lim }_{S^{\prime}}(\bar{A})_{m \in \mathbb{Z}} \cong(R / J(R))^{n} \otimes_{S^{\prime}} S^{\prime} \bar{A} \cong(R / J(R))^{n}(\bar{A}) .
\end{aligned}
$$

Since $Q \cong \bar{A}(R / J(R))^{n}$, we can conclude $N / J(R) N \cong \operatorname{Hom}_{R}(Q, R / J(R))$. As $N$ is a finitely generated, countably presented, flat module, this shows that (iv) and (v) hold.

By symmetry, that is, applying (i) implies (iv) and (v) to the opposite ring $R^{\mathrm{op}}$, we see that (iv) implies (i) and (ii). Hence (i), (ii), (iv) and (v) are equivalent statements.

By Proposition 6.3, (iii) implies (i). Assume that (ii) holds, so that there exist a finitely generated, countably presented, flat right $R$-module $M$ and an isomorphism $\alpha: M / M J(R) \rightarrow P$. The module $M$ has projective dimension $\leq 1$ [Lazard 1969, Théorème 3.2]. Let $\pi:(R / J(R))^{n} \rightarrow P$ be the projection with kernel $Q$. The onto mapping $\alpha^{-1} \pi:(R / J(R))^{n} \rightarrow M / M J(R)$ can be lifted to a homomorphism of right $R$-modules $\psi: R_{R}^{n} \rightarrow M_{R}$, which is necessarily onto by Nakayama's Lemma. As the conditions of Proposition 6.3(i) are satisfied, we deduce the existence of a right projective module $Q^{\prime}$ such that $Q^{\prime} / Q^{\prime} J(R) \cong Q$. This proves that (ii) implies (iii), so that (ii) and (iii) are equivalent statements. By symmetry, (v) and (vi) are also equivalent. 
Recall that a projective module $P$ is a direct sum of countably generated submodules, and that $P=0$ if and only if $P / P J(R)=0$. Hence, if a projective module is finitely generated modulo the Jacobson radical, it must be countably generated. Thus the modules $P^{\prime}$ and $Q^{\prime}$ in the statement of Theorem 7.1 are necessarily countably generated.

It would be interesting to know whether the module $M$ in Theorem 7.1(ii) is uniquely determined up to isomorphism. In Proposition 5.2 we saw an example of countably presented nonisomorphic cyclic flat modules that are isomorphic modulo the Jacobson radical, but the cyclic modules in that example are not projective modulo the Jacobson radical.

We conclude with two results related to this question, the first of which appears as [Lam 1999, p. 161, Exercise 20]. We give a proof for the sake of completeness.

Lemma 7.2. Let $M$ be a finitely generated flat right module over a ring $R$, and let $P$ be a projective right $R$-module. If $\gamma: P \rightarrow M$ is a projective cover, then $\gamma$ is an isomorphism.

Proof. The module $P$ is finitely generated because $M$ is finitely generated and ker $\gamma$ is small in $P$. Hence there exist $n$ and a projective module $Q$ such that $P \oplus Q \cong R^{n}$. As $\gamma \oplus 1_{Q}: P \oplus Q \rightarrow M \oplus Q$ is a projective cover, and $\gamma$ is an isomorphism if and only if so is $\gamma \oplus 1_{Q}$, we may assume without loss of generality that $P$ is $R^{n}$ and that $\gamma: R^{n} \rightarrow M$ is a projective cover.

Let $x \in \operatorname{ker} \gamma$. By Proposition 4.2, there exists $A \in M_{n}(R)$ such that $A x=x$ and $A R^{n} \subseteq \operatorname{ker} \gamma \subseteq R^{n} J(R)$. This implies that $(1-A) x=0$ and that $A \in M_{n}(J(R))$, thus $x=0$. This shows that $\operatorname{ker} \gamma=0$, hence $\gamma$ is an isomorphism.

Proposition 7.3. Let $M$ be a finitely generated flat right module over a ring $R$, and let $P$ be a projective module. If $M / M J(R) \cong P / P J(R)$, then $M \cong P$.

Proof. As $M / M J(R) \cong P / P J(R)$, the module $P / P J(R)$ is finitely generated. We will prove that $P$ is, in fact, finitely generated.

By Theorem 7.1, there exists a finitely generated, countably presented, flat module $M^{\prime}$ such that $P / P J(R) \cong M^{\prime} / M^{\prime} J(R)$. Let $\alpha: P / P J(R) \rightarrow M^{\prime} / M^{\prime} J(R)$ be an isomorphism. Let $\pi: P \rightarrow P / P J(R)$ and $\pi^{\prime}: M^{\prime} \rightarrow M^{\prime} / M^{\prime} J(R)$ denote the canonical projections. Since $P$ is projective, there exists $\beta: P \rightarrow M^{\prime}$ such that the diagram

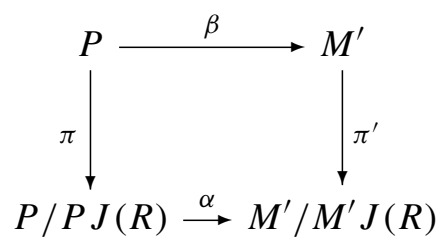


is commutative. Since $\operatorname{ker} \pi^{\prime}$ is small in $M^{\prime}, \beta$ is onto. As $M^{\prime}$ has projective dimension $1, \operatorname{ker} \beta$ is projective. Applying $-\otimes_{R} R / J(R)$ to the exact sequence

$$
0 \longrightarrow \operatorname{ker} \beta \rightarrow P \stackrel{\beta}{\longrightarrow} M^{\prime} \longrightarrow 0,
$$

we obtain the exact sequence

$$
0 \longrightarrow \operatorname{ker} \beta \otimes_{R} R / J(R) \rightarrow P / P J(R) \stackrel{\alpha}{\longrightarrow} M^{\prime} / M^{\prime} J(R) \longrightarrow 0 .
$$

Since $\alpha$ is an isomorphism, we have $0=\operatorname{ker} \beta \otimes_{R} R / J(R) \cong \operatorname{ker} \beta /(\operatorname{ker} \beta) J(R)$. But $\operatorname{ker} \beta$ is projective, hence $\beta$ is an isomorphism. This proves that $P$ is a finitely generated projective module.

Let $\rho: M \rightarrow M / M J(R)$ denote the canonical projection. Since $P$ is projective, there exists $\gamma: P \rightarrow M$ such that the diagram

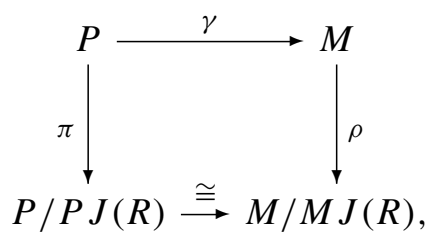

is commutative. Since $\operatorname{ker} \rho$ is small in $M, \gamma$ is onto. Since $P$ is finitely generated and $\operatorname{ker} \gamma \subseteq P J(R), \operatorname{ker} \gamma$ is small in $P$. Hence $\gamma: P \rightarrow M$ is a projective cover. By Lemma 7.2, $\gamma$ is an isomorphism.

Thus if a finitely generated projective right $R / J(R)$-module $P$ satisfies condition (i) of Theorem 7.1 (that is, $P \cong M / M J(R)$ for some finitely generated flat right $R$-module $M$ ) and the right/left symmetric of condition (vi) of Theorem 7.1 (that is, $P \cong P^{\prime} / P^{\prime} J(R)$ for some projective right $R$-module $P^{\prime}$ ), then $M \cong P^{\prime}$ is a projective cover of $P$.

\section{Acknowledgement}

We are grateful to G. Puninski for his comments on a previous version of this paper.

\section{References}

[Anderson and Fuller 1992] F. W. Anderson and K. R. Fuller, Rings and categories of modules, 2nd ed., Graduate Texts in Mathematics 13, Springer, New York, 1992. MR 94i:16001 Zbl 0765.16001 [Azumaya 1987] G. Azumaya, "Finite splitness and finite projectivity", J. Algebra 106:1 (1987), 114-134. MR 89a:16034 Zbl 0607.16017

[Bass 1960] H. Bass, "Finitistic dimension and a homological generalization of semi-primary rings", Trans. Amer. Math. Soc. 95 (1960), 466-488. MR 28 \#1212 Zbl 0094.02201

[Jøndrup 1976] S. Jøndrup, “Projective modules”, Proc. Amer. Math. Soc. 59:2 (1976), 217-221. MR 54 \#7546 Zbl 0338.16008 
[Lam 1999] T. Y. Lam, Lectures on modules and rings, Graduate Texts in Mathematics 189, Springer, New York, 1999. MR 99i:16001 Zbl 0911.16001

[Lazard 1969] D. Lazard, “Autour de la platitude”, Bull. Soc. Math. France 97 (1969), 81-128. MR 40 \#7310 Zbl 0174.33301

[Lazard 1974] D. Lazard, "Liberté des gros modules projectifs", J. Algebra 31 (1974), 437-451. MR 50 \#2250 Zbl 0291.13004

[Puninski 2004] G. Puninski, "Projective modules over the endomorphism ring of a biuniform module”, J. Pure Appl. Algebra 188:1-3 (2004), 227-246. MR 2004k:16003

[Sakhaev 1987] I. I. Sakhaev, “On the group $K_{0}(A \Pi)$ for semilocal rings", Math. Nachr. 130 (1987), 157-175. MR 88e:18010 Zbl 0617.13009

[Sakhaev 1993] I. I. Sakhaev, "Finite generability of projective modules over rings with polynomial identities", Izv. Vyssh. Uchebn. Zaved. Mat. 8 (1993), 65-75. In Russian; translated in Russian Math. (Iz. VUZ) 37 (1993), 64-74. MR 95k:16004

[Sakhaev 1996] I. I. Sakhaev, "Finite generation of projective modules over some rings", Izv. Vyssh. Uchebn. Zaved. Mat. 10 (1996), 63-75. In Russian; translated in Russian Math. (Iz. VUZ) 40 (1997), 60-72. MR 98e:16004

[Vasconcelos 1969] W. V. Vasconcelos, "On projective modules of finite rank", Proc. Amer. Math. Soc. 22 (1969), 430-433. MR 39 \#4134 Zbl 0176.31601

[Zöschinger 1981] H. Zöschinger, "Projektive Moduln mit endlich erzeugtem Radikalfaktormodul", Math. Ann. 255:2 (1981), 199-206. MR 82e:16013 Zbl 0439.16015

Received July 11, 2003. Revised July 13, 2004.

\section{ALbERTO FACCHINI \\ Dipartimento di Matematica PuRa ed ApPlicata \\ UNIVERSITÀ DI PADOVA \\ 35131 PADOVA}

ITALY

facchini@math.unipd.it

http://www.math.unipd.it/ facchini

\section{DOLORS HERBERA}

DePartament DE MATEMÀtiques

UNIVERSITAT AUTÒNOMA DE BARCELONA

08193 BELLATERRA (BARCELONA)

SPAIN

dolors@mat.uab.es

ISKHAK SAKHAJEV

DePaRTMENT OF MECHANiCS AND MATHEMATICS

KAZAN'S UNIVERSITY OF TATARSTAN

420008 KAZAN

RUSSIA

ishak.sahajev@ksu.ru 